Review Article

\title{
Undersized Indian Children: Nutrients-starved or Hungry for Development?
}

\author{
H P S SACHDEV* \\ Senior Consultant Pediatrics and Clinical Epidemiology, Sitaram Bhartia Institute of Science and Research, \\ B-16 Qutab Institutional Area, New Delhi 110 016, India
}

(Received on 22 May 2018; Accepted on 13 June 2018)

\begin{abstract}
Undersized children ( $<5$ years) have body dimensions $<-2$ standard deviation, SD, of WHO Multicentre Growth Reference, constructed from affluent subjects with no health or environmental constraints. Lower anthropometric measurements portray only undersize irrespective of cause, but such children are labelled as undernourished: underweight (low weight; weight-for-age <-2SD), stunted (short child; height-for-age <-2SD), and/or wasted (thin child; weight-for-height <-2SD). Undersize in children is actually a crude proxy for overall socio-economic underdevelopment and intergenerational constraint of maternal undersize, but it has paradoxically come to be perceived as a proxy for nutritional inadequacy in both mainstream and under-privileged populations. Evidence indicates that only some nutrients or food-based interventions, including those during pregnancy, increase anthropometric indices, and those too do so only modestly (0.1-0.25 SD or 5\%$10 \%$ deficit) with unsustainable benefits. Other determinants include water, sanitation and hygiene; nutrition counselling; maternal characteristics; curative and preventive health care; maternal, household and community resources; literacy; income; women empowerment; safety nets; and genes. In tandem with the overall national development, time trends show a gradual decline in the incidence of undersize in children; this trend has hastened in the past decade, even amongst the underprivileged. The steady decline in the prevalence of stunting and underweight, with wasting levels remaining stagnant, is observed in neighbouring countries too and appears unrelated to specific determinants. Biological and population transition evidence suggests that rapid and simultaneous declines in both stunting and wasting are challenging to achieve. Patience is required to overcome the legacy of considerable intergenerational handicap. A narrow focus on nutrients-based solutions will fail to accelerate progress. Comprehensive and equitable development is the key to success. With substantial earlier investments in dietary subsidies, action is now warranted in other sectors to rapidly benefit the marginalised.
\end{abstract}

Keywords: Anthropometry; Development; Nutrients; Stunting; Under-five Children; Undernutrition; Undersize; Underweight; Wasting

\section{Introduction}

For centuries, a good adult physique has symbolised strength, health, affluence and smartness in mankind. Good-sized children herald an enviable adult physique, and also epitomise these attributes. Populations therefore covet this characteristic and justifiably endeavour to address undersize in children. This evidence-informed communication explores the ingredients for accelerating progress in this direction in India. The probe is topical and timely because of the ongoing debate on recasting the nutrition mission, given that the massive investment in food subsidies and food supplementation have not substantially improved the situation.

\section{Reference for Defining Undersize}

Children below five years of age with body dimensions below a statistically defined cut-off are classified as undersized. This cut-off is minus two (-2) standard deviations (SD) of analogous reference charts for that age and gender. If 100 children of a given age and sex are arranged in an ascending order of height or weight, then the cut-off of -2SD roughly corresponds to the $3^{\text {rd }}\left(2.5^{\text {th }}\right)$ shortest or lightest child. The World 
Health Organisation's (WHO) Multicentre Growth Reference Study (MGRS) charts serve as the global reference (WHO Multicentre Growth Reference Study Group, 2006a), but are invariably being used as a standard. The distinction between these two terms is fundamental because a reference is for comparison only without ascribing the worth, whereas; a standard implies a valuable target to be achieved. Few would argue about the need to passionately pursue this standard; however, this aspiration must be balanced with realistic expectations.

The MGRS (July 1997-December 2003) was a population-based study carried out in the cities of Davis, California, USA; Muscat, Oman; Oslo, Norway; and Pelotas, Brazil; and in selected affluent neighbourhoods of Accra, Ghana, and South Delhi, India (WHO Multicentre Growth Reference Study Group, 2006a). The study populations lived in socioeconomic conditions favourable to growth and where mobility was low, $>20 \%$ of mothers followed WHO feeding recommendations and breastfeeding support was available. Individual inclusion criteria were: no known health or environmental constraints to growth, mothers willing to follow MGRS feeding recommendations (exclusive or predominant breastfeeding for at least four months, introduction of complementary foods by the age of six months and continued partial breastfeeding up to at least 12 months), no maternal smoking before and after delivery, single term birth and absence of significant morbidity. Only $17 \%$ of the screened newborns were eligible for inclusion.

The prescriptive approach taken in developing these references is thus fundamentally different from the earlier descriptive references based on geographically representative samples of children, regardless of feeding or other behaviours. This prescriptive approach describes the growth of children who are free from socio-economic, environmental and biological constraints, and whose care has followed recommended health practices and behaviours associated with healthy outcomes. Although neither biomarkers nor nutrient intakes were quantified, these children were presumed to be nutritionally adequate because of the eligibility criteria. Thus low anthropometric values in MGRS subjects would not arise from nutritional compromise but may result from biologic variability or additional unmeasured factors (for example, rare hormonal disorders).

Across the six sites, there was a striking correspondence of measurements; similarity was defined a priori as site differences below $0.5 \mathrm{SD}$, but these were usually below $0.2 \mathrm{SD}$. This suggested that under optimal conditions, there is negligible contribution of genetic variation to childhood growth (WHO Multicentre Growth Reference Study Group, 2006b). The WHO MGRS growth curves are therefore expected to provide a single international reference that represents the best description of physiological growth for all children below five years of age and to establish the breastfed infant as the model for growth and development. However, currently only 5-10\% of Indian children fulfil the WHO MGRS eligibility criteria, primarily due to co-existence of several exclusion factors.

\section{Leap of Faith}

Notwithstanding the fact that lower anthropometric values portray an undersized individual irrespective of the cause, the prevailing nomenclature for such children is undernourished. Using WHO MGRS reference, childhood "undernutrition" in populations is conventionally measured by three anthropometric indices: underweight (low weight; weight-for-age <2SD), stunted (short child; length or height-for-age $<-2 \mathrm{SD}$ ), and wasted (thin child; weight-for-length or height $<-2 \mathrm{SD}$ ). A wasted child is considered to have acute undernutrition, stunting represents a longer lasting deprivation and weight for age is a composite measure of these two indices.

Considering the stringent eligibility criteria for constructing the WHO reference, what does the prevalence of undersized children represent at population level? It is a crude but convenient proxy for a blend of overall socio-economic development and intergenerational constraint of maternal undersize. Nutrients deficits, particularly in individuals, may comprise only one component of this multi-faceted surrogate. However, paradoxically, for determining public health response, a reference for body size constructed from elite subjects has primarily become a standard of nutritional adequacy for both mainstream and underprivileged populations. Pertinent data indicate that this extrapolation of a reference into a standard applicable to substantially different settings is predominantly faith-based rather 
than evidence-based, because the available evidence is indirect and of very low quality by GRADE criteria (GRADE Working Group, 2004).

\section{Evidence Base}

The following overview principally addresses stunting because it is currently believed to be the best predictor for physical, neuro developmental and economic capacity, and possibly an indicator of elevated risk of cardio-metabolic risk in adulthood (Victora et al., 2008). However, the findings are also largely applicable to the other two indices - wasting and underweight.

\section{Food and Nutrients-based Solutions}

Experimental designs (for example, randomized controlled trials) provide robust estimates of the best expectations from proposed interventions. Such evidence indicates that only some nutrients-based or food-based interventions, including those during pregnancy, improve anthropometric readings, and even with those the impact is quite modest, generally ranging between 0.1 and 0.25 SD or roughly $5-10 \%$ of the current deficit (Dewey and Adu-Afarwuah, 2008; Haider and Bhutta, 2017; Kristjansson et al., 2015; Lassi et al., 2013; Ramakrishnan et al., 2009). The Lancet Series on Nutrition modelled the population impact of ten such direct nutrition interventions for 2011 in 34 countries harbouring 90\% of the global burden of stunting (Bhutta et al., 2013). These "nutrient-specific" interventions included periconceptional folic acid supplementation or fortification, maternal balanced energy protein supplementation, maternal calcium supplementation, multiple micronutrient supplementation in pregnancy, promotion of breast feeding, appropriate complementary feeding, vitamin A and preventive zinc supplementation in children 6-59 months of age, management of severe acute malnutrition (severe wasting) and management of moderate acute malnutrition (moderate wasting). Scaling up of all ten interventions to $90 \%$ coverage was associated with only a mean $20.3 \%$ (range $10.2 \%$ to $28.9 \%$ ) reduction in stunting.

In our context, these are likely to be substantial overestimates from purely a nutrients supplementation perspective because (Sachdev et al., 2013): (i) achieving $90 \%$ coverage under programmatic conditions is very difficult, if not impossible; (ii) several assumptions and imputations were used, which are brittle and sensitive to alternative philosophies and effect size; (iii) a large negative trial of zinc supplementation was not included in the meta-analysis; (iv) counselling for breastfeeding promotion and complementary feeding was combined with food supplements for computations; (v) other non-"nutrientspecific" interventions were not simultaneously modelled thereby inflating projections; and (vi) there is uncertainty about sustainability of anthropometric benefit after discontinuation of supplementation (Devakumar et al., 2016; Trilok-Kumar G et al., 2015).

\section{Other Potential Determinants}

Improved water, sanitation and hygiene (WASH) practices over a prolonged period (9-12 months or more) may improve anthropometric readings slightly ( $\sim 0.08$ SD) or have no benefit (Dangour et al., 2013; Gera et al., 2018). Even in food-insecure populations, nutrition counselling of the mother has a similar or only marginally lower $(\sim 0.2 \mathrm{SD})$ effect in comparison to provision of complementary feeding in children below 2 years of age (Lassi et al., 2013). Ethical and logistic considerations preclude the gathering of experimental evidence for several non-nutrient-based solutions, also referred to as "nutrition-sensitive" interventions that address the underlying determinants of undersize (Prendergast and Humphrey, 2014; Ruel et al., 2013; Sachdev, 2012; Stewart et al., 2013). These mostly echo the eligibility criteria of WHO MGRS, encompassing maternal characteristics such as age at child bearing, spacing, pre-pregnant height and weight; access to curative and preventive health care services including immunisation and treatment during illnesses; adequate care-giving resources at maternal, household and community levels; literacy; level of women empowerment; and socio-economic status including safety nets. However, good quality observational evidence indicates that each of these factors, included in the broad canvas of "development", contributes to the body size of children. The quantum of effect varies in different contexts and is probably similar to or sometimes greater than isolated nutrients-based interventions. Body size is also a heritable trait, and over 200 genes have been identified in genome-wide association studies for adult height; however, together these explain only $10 \%$ of 
the variation (Berndt et al., 2013; Lango Allen et al., 2010).

\section{Misdirected Stigma}

The advocacy machinery tends to portray national child undersize statistics as "hunger" or "starvation" burden, and this is accordingly sensationalised by the lay media. Is this not a misdirected stigma for a maldevelopment process? Our nation not only has an ongoing epidemic of over-nutrition-associated noncommunicable diseases but has also achieved selfsufficiency in food production decades ago with an occasional paradox of rotting of excess food stocks. A similar stigma is often experienced by undersized individuals consuming sufficient food! Apart from the avoidable disdain, a misdirected stigma can only result in an inappropriate response.

\section{Contemporary Public Health Response}

Food and nutrients subsidies or supplementation are the most popular and often the only strategy for addressing population undersize. Politicians, other stakeholders and beneficiaries are invariably mesmerised by this approach because: (i) there is a deep rooted socio-cultural belief, persisting from the famine era, that children's body size is directly proportional to food intake; (ii) a visible intervention testifies sensitivity of governance and signals responsiveness to recipients; (iii) finances earmarked for food in the household budgets of the beneficiaries become available for other necessities; (iv) targeted advocacy, often catalysed by the food and nutrients industry, promises highly exaggerated benefits; this amounts to commercialisation of undernutrition; and (v) the corrupt can use it as a convenient conduit to siphon off the public money.

Undeniably, there is a definite role of food or nutrients distribution during disaster or famine situations. Further, there is merit in the assertion that a resource-starved country cannot afford all interventions simultaneously and providing food to the poor and marginalised is a noble beginning, addressing the basic human necessity and proximal cause of undersize. However, this approach necessitates tolerating the following undesirable consequences: (i) insufficient benefits from this partial intervention; (ii) complacence among stakeholders that everything possible is being done; (iii) diversion of public finances and attention from other crucial unattended determinants of undersize; (iv) dependence by the beneficiaries on public welfare measures for daily existence; and (v) the risk that long-term food-based supplementation (primarily cereals) can fuel the ongoing epidemic of associated non-communicable diseases (diabetes, hypertension and coronary heart disease) even among the poor and undersized (Leroy et al., 2013).

In global nomenclature, undersized children are considered to be 'undernourished'. Predictably, therefore, most stakeholders, particularly the policy makers and civil society, attribute undersize to nutrients-deprivation and focus public health efforts towards food or nutrient-product based solutions. Consequentially, other important determinants, outlined earlier, are seldom visualised or actively targeted for improving undersize. However, efforts have now been initiated for amalgamation with WASH interventions. In addition, other important themes contributing to the current status need priority consideration in order to accelerate the pace of improvement.

\section{Scope for Improvement: Practical Considerations}

The prevalent situation and pace of improvement are invariably assessed in terms of a national average, often viewed in isolation from additional relevant information. Attempts to accelerate the pace of progress must also consider the following: (i) Inequity: The inequity gap persists and often widens (Bredenkamp et al., 2014; Kumar et al., 2015); the greater the proportion of the population that is deprived, the higher is the burden and slower the improvement. (ii) Comprehensive interventions: Isolated interventions (for example, only nutrients supplementation or only sanitation) yield fractional benefits with partial leakage from gaps created by other determinants; intuitively an all-inclusive approach should maximize the rewards. (iii) Windows of opportunity: Policy makers and stakeholders, particularly program managers, find it attractive and pragmatic to restrict interventions to "vulnerable" segments of the life cycle for "maximum impact". The current focus on 1000 days beginning from conception has gained acceptance because a substantial part of subsequent growth trajectory is 
established within two years of birth (Bhutta et al., 2013; Prendergast and Humphrey, 2014; Sachdev, 2012). Nevertheless, in a real-life scenario, insults and transitions in anthropometry occur throughout the life span. Importantly, adolescence is an often ignored but promising period with benefits spilling over to the next generation (Patton et al., 2018). (iv) Sustainability: Solutions need to be sustainable, ideally over generations, because interruptions stop or hinder progress. (v) Baseline: Populations starting at lower levels invariably take longer to reach the target. (vi) Intergenerational handicap: More than one generation may be needed to wipe out the remnants of adversities experienced by ancestors (for example, maternal height - a marker of biological constraint); only a limited improvement is possible within one generation (Martorell and Zongrone, 2012).

\section{Time Trends}

There exists a clear evidence of increase in body size over the past four decades even among the underprivileged (Paul et al., 2011; Sachdev and Shah, 2007; Sachdev, 2012). The time trends in underweight, stunting and wasting from successive National Family Health Surveys are graphically summarized in Fig. 1. These improvements antedated the introduction of food subsidies, and progressed at a somewhat faster pace during the past decade. A quantification of the progress rate is difficult because of variations in sampled areas, age groups, and cut-offs to define undersize. However, a reasonable comparison is feasible in under-five children on the basis of data from the last two National Family Health Surveys, the NFHS-3 in 2005-06 and

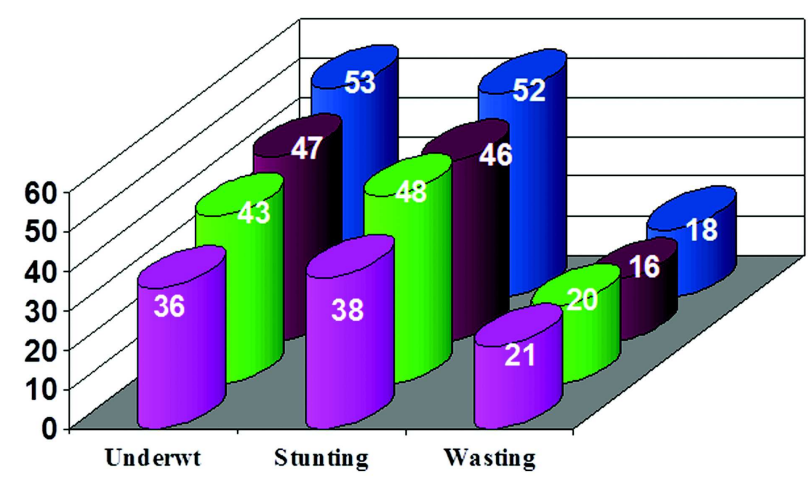

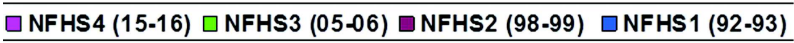

Fig. 1: Time trend in prevalence (\%) of underweight, stunting and wasting from the successive National Family Health Surveys the NFHS-4 in 2015-16 (International Institute for Population Sciences and Macro International, 2007; International Institute for Population Sciences, 2016). Over 10 years, stunting declined from $48 \%$ to $38.4 \%$ (9.6\%) and underweight from $42.5 \%$ to $35.7(6.8 \%)$, while wasting increased marginally from $19.8 \%$ to $21 \%(1.2 \%)$. The corresponding relative declines for stunting and underweight were $20 \%$ and $16 \%$, respectively. This transition pace should instil optimism and not despair, as projected by current national comparisons, without consideration of earlier estimates at similar time points. For example, the stunting prevalence in Brazil four decades ago (197475 ) was $37.1 \%$; marginally lower $(1.7 \%)$ than our national estimates today (Monteiro et al., 2010). In India, the relatively greater decline in stunting is encouraging because improvements in length are more difficult to achieve than increases in weight.

These improvements occurred in tandem with the overall developmental process, a small component of which was food subsidies. This inference is supported by intergenerational comparison, over the past 30 years, of anthropometric data of children and their parents in the New Delhi Birth Cohort subjects from middle socio-economic status who were not recipients of food subsidies (Sinha et al., 2017). In comparison to their parents, children were considerably taller $(0-5$ years $0.99 \mathrm{SD} ; 5-10$ years $1.17 \mathrm{SD})$ and heavier ( $0-5$ years $0.77 \mathrm{SD}$; $5-10$ years $1.52 \mathrm{SD}$ ) while only those aged 5-10 years were broader (had a higher BMI; 1.03 SD). These increases for 0-5 and 5-10 years, respectively corresponded to 3.9 and $6.4 \mathrm{~cm}$ for height, 1.3 and $5.4 \mathrm{~kg}$ for weight and 0.2 and $1.9 \mathrm{~kg} / \mathrm{m}^{2}$ for BMI. Greater intergenerational gains in children were associated with lower anthropometric indices in parents, and in children (when measured), poor water supply and sanitation facilities, higher age of parents at child birth (for height and weight); and more parental education (for weight and BMI). These improvements are a crude approximation of what is achievable over just one generation even with modest developmental constraints. The intergenerational changes for height in the New Delhi Birth Cohort are substantially greater than those observed in the UK - at 7 years, offspring were only slightly taller $(0.19 \mathrm{SD}$ or $1 \mathrm{~cm})$ than their parents (Li and Power, 2004). 


\section{Stagnant Wasting Levels}

Understandably, stagnant wasting levels (Fig. 1) are disconcerting for the stakeholders, particularly because of application of the terms 'moderate' or 'severe acute' malnutrition for moderate or severe wasting, respectively. These terminologies, employed for thinness and severe thinness, evoke perceptions of "hunger" and "starvation", and life-threatening emergencies. Recent DHS surveys suggest a similar pattern of decline in stunting prevalence and stagnant wasting prevalence in the neighbouring countries.

The underlying and proximate factors related to stunting and wasting are similar (Martorell and Young, 2012). A steady improvement in stunting and underweight with stagnant wasting levels, is therefore unlikely to be related to non-improvement of specific determinants like food or nutrients. This is probably a reflection of biological thinness (thin-fat infant phenotype) of Indian populations (Yajnik et al., 2003) or the pattern of anthropometric change in stunted populations undergoing nutrition transition. In underfive children, increase in length (or height) and breadth are two distinct biological processes, which generally do not occur simultaneously. Longitudinal data in under-five children from the New Delhi Birth Cohort (Fig. 2) suggest that height-for-age and weight-forheight have an inverse relationship-that is, with higher prevalence of stunting, the prevalence of wasting declines. Further, evidence including that from India, indicates that children "grow up" (get taller) before "growing out" (getting broader or increasing BMI) (Sinha et al., 2017; Smith et al., 2013). This observation is also consistent with the increased prevalence of obesity in older children from India (Hoque et al., 2014), and other countries (Moon, 2011; Smith et al., 2013; Zong and Li, 2014). The underlying mechanisms for this phenomenon are unclear. Thus, rapid and simultaneous declines in both stunting and wasting prevalences, as perceived in the sustainable development goals, appear challenging, if not impossible, to achieve.

\section{Moving Target}

Contemporary evidence of time trends suggests that, also in high-income and well-developed countries, body size continues to increase (NCD Risk Factor Collaboration, 2016), even in children. Thus additional unknown drivers are fuelling the biological processes to attain greater body size. It is likely that the WHO reference would shift up if a similar study is conducted today or a decade later. This upward movement of the target will naturally increase the undersize burden. It would be important to remember that our real goal is to minimize the gap between the "best" and "others"; that is, to achieve equity.

\section{Accelerating Progress}

Transparent, undisputed and effective communication of diagnoses and remedies is crucial. Politicians, policy makers, other stakeholders and the lay public must
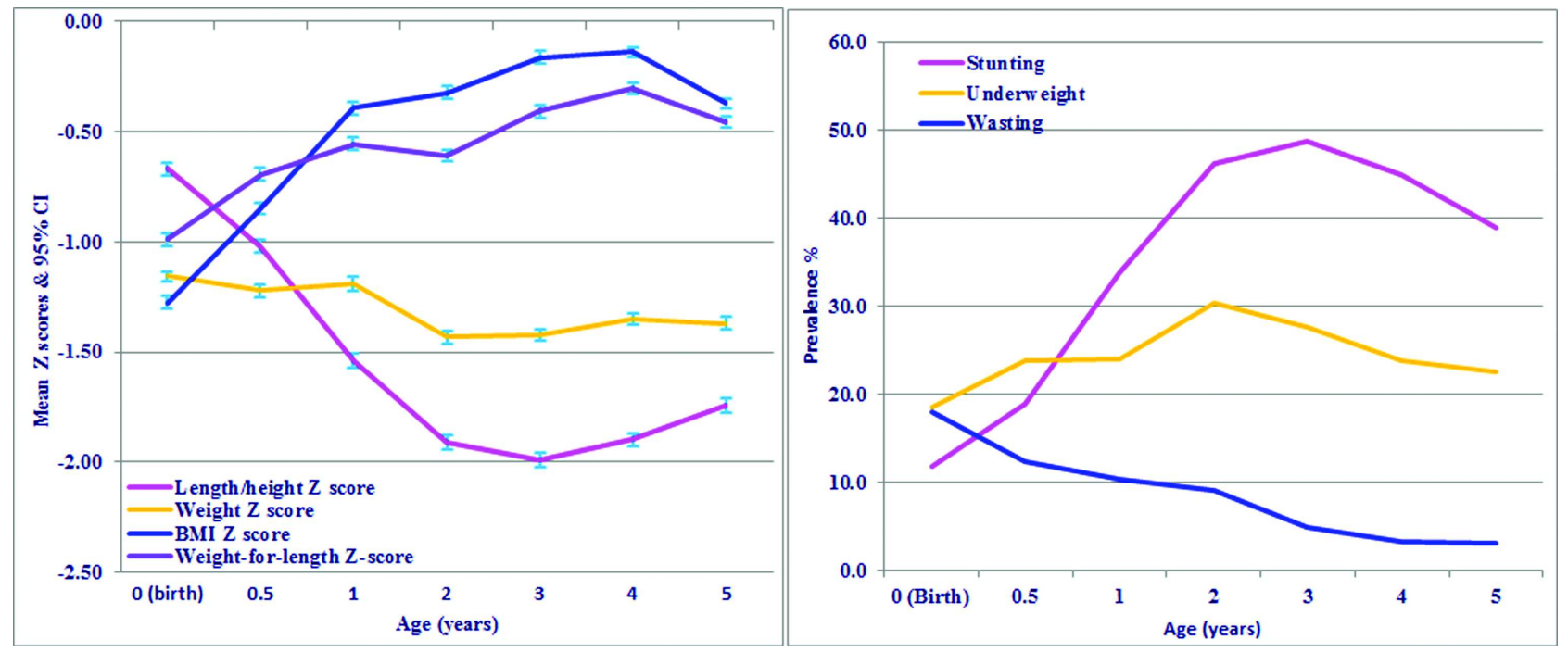

Fig. 2: Longitudinal changes in anthropometric indices and prevalence (\%) of underweight, stunting and wasting in underfive children from the New Delhi Birth Cohort 
realize that there are no magical solutions to wipe away the problem of undersize in children. Undersize reflects wider societal maldevelopment and inequity; isolated nutrients-starvation has a fairly limited role. Progress can be accelerated only by making interventions comprehensive (simultaneously addressing all determinants) and inclusive (prioritising the deprived). Focussing solely on nutrients and 1-2 additional determinants (for example, WASH interventions) will yield leaky, slow and disappointing results.

The academia, the research community and traditional advocates, have clearly recognized the root malady for over two decades; the responsibility for communicating these rests with them .However, it was only in 2013 that the term 'nutrition-sensitive interventions' was coined to explore the contributions of underlying determinants (Ruel et al., 2013). Also, in November 2014, after the launch of "Swach Bharat Abhiyan" by the government, sanitation is being propagated as an additional thrust intervention. It remains an enigma though, that credible advocates continue to synonymise undersize with undernutrition, focus on nutrients-based (nutrient specific) solutions and relegate other important determinants to the background.

The potential reasons, including those based on rumour, include: (i) A dogmatic belief that all underlying factors will operate though proximal determinants, primarily nutrients, and that managing childhood illnesses is health sector's domain; (ii) marketing/promoting a comprehensive and inclusive development agenda is challenging; it is too diffuse, unmanageable and unattractive whereas nutrients- or product-based solutions are tangible and appealing; (iii) a pragmatic balance is being maintained between the optimal and feasible when a noble interim intervention with no harms is made. Added to these are commercial factors: (i) the nutrients-based sector's prominence and continued funding lead to stability in careers and institutions; and (ii) industrial nudging for sharing profits.

Advocacy must rise above restricted considerations and instil realism, leading to appropriate expectations and action. A narrow focus should not impede comprehensive and inclusive development. Irrespective of the benefits on body size, every ingredient of the development process deserves to be in place in its own right; for example, purchasing power; access to education, health care, water supply and sanitation services; and nutritional security. A seemingly nebulous agenda can be made more finite and structured by coalescing 2-3 key indicators each from the above key domains to monitor progress and fine-tune interventions. Efforts in this direction are beginning to surface.

Adequate funding is indispensable for public welfare. A policy based principally on continuing nutrients subsidies is economically challenging, yields disappointing results, and starves resources for other facets of development. Conversely, national economic growth alone may not automatically improve body size. Accelerated progress can occur only by ensuring direct investments for pro-poor development, particularly for education, health care, water supply and sanitation services. Substantial expenditure is already being incurred for nutrients subsidies. In the oft-cited role model of the Brazilian experience, socioeconomic development coupled with equity-oriented policies, and not dietary subsidies, yielded noteworthy results (Monterio et al., 2010). The poor have a right to be equal partners in sustained development instead of continuing to subsist on food hand-outs.

\section{Concluding Comments}

Undersized Indian children have a legacy of considerable inter-generational handicap necessitating a greater catch-up period even under the best circumstances; we therefore need to be patient and practical. There has been a gradual improvement, which has hastened in the past decade. A predominant focus on nutrients-based solutions will fail to accelerate progress. Comprehensive and equitable development is the key to success. The nation has already invested heavily in dietary subsidies, setting the stage for an urgent and greater focus on other sectors which will rapidly benefit the poor and marginalised. 


\section{References}

Berndt S I, Gustafsson S, Magi R, Ganna A, Wheeler E, Feitosa M F, et al. (2013) Genome-wide meta-analysis identifies 11 new loci for anthropometric traits and provides insights into genetic architecture Nat Genet 45 501-512

Bhutta Z A, Das J K, Rizvi A, Gaffey M F, Walker N, Horton S, Webb P, Lartey A, Black R E (2013) The Lancet Nutrition Interventions Review Group, and the Maternal and Child Nutrition Study Group. Evidence-based interventions for improvement of maternal and child nutrition: what can be done and at what cost? Lancet 382 452-477

Bredenkamp C, Buisman L R and Ellen Van de Poel (2014). Persistent inequalities in child undernutrition: evidence from 80 countries, from 1990 to today Int J Epidem $\mathbf{4 3}$ 1328-1335

Dangour AD, Watson L, Cumming O, Boisson S, Che Y, Velleman Y, Cavill S, Allen E and Uauy R (2013) Interventions to improve water quality and supply, sanitation and hygiene practices, and their effects on the nutritional status of children Cochrane Database of Systematic Reviews Issue 8. Art. No.: CD009382. DOI: 10.1002/14651858. CD009382.pub2

Devakumar D, Fall C H D, Sachdev H S, Margetts B M, Osmond C, Wells J C K, Costello A and Osrin D (2016) Maternal antenatal multiple micronutrient supplementation for longterm health benefits in children: a systematic review and meta-analysis BMC Medicine 1490 DOI 10.1186/s12916016-0633-3

Dewey K G and Adu-Afarwuah S (2008) Systematic review of the efficacy and effectiveness of complementary feeding interventions in developing countries Mat Child Nutr $\mathbf{4}$ 24-85

Gera T, Shah D and Sachdev H S (2018) Impact of Water, Sanitation and Hygiene interventions on growth, Non-diarrheal morbidity and mortality in children residing in Low- and Middle-income Countries: A systematic review Indian Pediatr 55 381-393

Grades of Recommendation, Assessment, Development, and Evaluation (GRADE) Working Group (2004) Grading quality of evidence and strength of recommendations $B M J$ 3281 490-1494

Haider B and Bhutta Z A (2017) Multiple-micronutrient supplementation for women during pregnancy. Cochrane Database Syst Rev 4 CD004905 doi:10.1002/ 14651858.CD004905.pub5

Hoque M E, Doi S A, Mannan M, Long K, Niessen L W and Mamun AA (2014) Prevalence of overweight and obesity among children and adolescents of the Indian subcontinent:
A meta-analysis Nutr Rev 72 541-550

International Institute for Population Sciences (IIPS) and Macro International (2007) National Family Health Survey (NFHS-3), 2005-06: India: Volume I. Mumbai: IIPS. Available from http://www.nfhsindia.org/NFHS3\%20Data/VOL-1/India_volume_I_corrected_17oct08. pdf. Accessed on May 14, 2018

International Institute for Population Sciences (2016) National Family Health Survey (NFHS-4), 2013-14. Factsheet. Available from: http://rchiips.org/NFHS/factsheet_NFHS4.shtm. Accessed on May 14, 2018

Kristjansson E, Francis D, Liberato S, Greenhalgh T, Welch V, Jandu M B, Batal M, Rader T, Noonan E, Janzen L, Shea B, Wells G A and Petticrew M (2015) Supplementary feeding for improving the physical and psychosocial health of disadvantaged infants and children aged three months to five years: what works and why? A combined systematic and realist review, 3ie Grantee Final Review. London: International Initiative for Impact Evaluation (3ie)

Kumar A, Kumari D and Singh A (2015). Increasing socioeconomic inequality in childhood undernutrition in urban India: trends between 1992-93, 1998-99 and 2005-06 Health Policy Plan 30 1003-16

Lango Allen H, Estrada K, Lettre G, Berndt S I, Weedon M N, Rivadeneira F, et al. (2010) Hundreds of variants clustered in genomic loci and biological pathways affect human height Nature 467 832-838

Lassi Z S, Das J K, Zahid G, Imdad A and Bhutta Z A (2013) Impact of education and provision of complementary feeding on growth and morbidity in children less than 2 years of age in developing countries: a systematic review BMC Public Health $13 \mathrm{~S} 13$

Leroy J L, Gadsden P, González de Cossío T and Gertler P (2013) Cash and in-kind transfers lead to excess weight gain in a population of women with a high prevalence of overweight in rural Mexico J Nutr 143 378-83

Li L and Power C (2004) Influences on childhood height: comparing two generations in the 1958 British birth cohort Int J Epidemiol 33 1320-1328

Martorell R and Young M F (2012) Patterns of stunting and wasting: potential explanatory factors Adv Nutr 3 227233

Martorell R and Zongrone A (2012) Intergenerational influences on child growth and undernutrition Paediatr Perinat Epidemiol 26 302-314

Monteiro C A, D'Aquino Benicio M H, Conde W L, Konno S, Lovadino A L, Barros A J D and Victora C G (2010) Narrowing socioeconomic inequality in child stunting: The 
Brazilian experience, 1974-2007 Bull World Health Organ 88 305-311

Moon J S (2011) Secular trends of body sizes in Korean children and adolescents: From 1965 to 2010 Korean J Pediatr 54 436-442

NCD Risk Factor Collaboration (2016) A century of trends in adult human height eLife 5 e13410

Patton G C, Olsson C A, Skirbekk V, Saffery R, Wlodek M E, Azzopardi P S, Stonawski M, Rasmussen B, Spry E, Francis K, Bhutta Z A, Kassebaum N J, Mokdad A H, Murray C J L, Prentice A M, Reavley N, Sheehan P, Sweeny K, Viner R M and Sawyer S M (2018) Adolescence and the next generation Nature 554 458-466

Paul V K, Sachdev H S, Mavalankar D, Ramachandran P, JeevaSankar M, Bhandari N, Sreenivas V, Sundararaman T, Govil D, Osrin D and Kirkwood B (2011). Reproductive health, and child health and nutrition in India: meeting the challenge Lancet 377 332-349

Prendergast A J and Humphrey J H (2014) The stunting syndrome in developing countries Paediatrics and International Child Health DOI 10.1179/2046905514Y.0000000158

Ramakrishnan U, Nguyen P and Martorell R (2009) Effects of micronutrients on growth of children under $5 \mathrm{y}$ of age: Meta-analyses of single and multiple nutrient interventions Am J Clin Nutr 89 191-203

Ruel M T, Alderman H and the Maternal and Child Nutrition Study Group (2013) Nutrition-sensitive interventions and programmes: how can they help to accelerate progress in improving maternal and child nutrition? Lancet 382 536551

Sachdev H P S and Shah D (2007) Epidemiology of maternal and fetal malnutrition in South Asia. In: Perinatal and Newborn Care in South Asia (Ed. Bhutta Z A) pp.75-105, Oxford University Press

Sachdev H P S (2012) Overcoming challenges to accelerating linear growth in Indian children Indian Pediatr 49 271-275

Sachdev H P S, Gupta A, Kapil U, Choudhury P, Dubey A P, Shiva M and Bansal C P (2013) Policy Implications of Lancet Series on Nutrition, 2013: Guard against Commercial Exploitation of Malnutrition. Accessed from World Public Health Nutrition Website http://wphna.org/ open-statement-from-india-on-the-lancet-series-onnutrition-2013/on November 30, 2014

Sinha S, Aggarwal A R, Osmond C, D Fall C H, Bhargava S K and Sachdev H S (2017) Intergenerational change in anthropometric indices and their predictors among children in New Delhi Birth Cohort Indian Pediatr 54 185-192

Smith S M, Craig L C, Raja A E, McNeill G and Turner S W (2013) Growing up before growing out: secular trends in height, weight and obesity in 5-6-year-old children born between 1970 and 2006 Arch Dis Child 98 269-273

Stewart C P, Iannotti L, Dewey K G, Michaelsen K F and Onyango A W (2013) Contextualising complementary feeding in a broader framework for stunting prevention Maternal and Child Nutrition 9 27-45

Trilok-Kumar G, Kaur M, Rehman A M, Arora H, Rajput M M, Chugh R, Kurpad A, Sachdev H S and Filteau S (2015) Effects of vitamin D supplementation in infancy on growth, bone parameters, body composition and gross motor development at age 3-6 years: follow-up of a randomized controlled trial Int J Epidemiol 44 894-905

Victora C G, Adair L, Fall C, Hallal P C, Martorell R, Richter L, Sachdev H S and Maternal and Child Undernutrition Study Group (2008) Maternal and child undernutrition: Consequences for adult health and human capital Lancet $371340-357$

WHO Multicentre Growth Reference Study Group (2006a) Enrolment and baseline characteristics in the WHO Multicentre Growth Reference Study Acta Pcediatrica 450 $7-15$

WHO Multicentre Growth Reference Study Group (2006b) Assessment of differences in linear growth among populations in the WHO Multicentre Growth Reference Study Acta Pcediatrica $\mathbf{4 5 0}$ 56-65

Yajnik C S, Fall C H D, Coyaji K J, Hirve S S, Rao S, Barker D J P, Joglekar C and Kellingray S (2003) Neonatal anthropometry: the thin-fat Indian baby. The Pune Maternal Nutrition Study Int J Obes Relat Metab Disord 27 173-80

Zong X N and Li H (2014) Physical growth of children and adolescents in China over the past 35 years Bull World Health Organ 92 555-564. 